\title{
Chemical composition of chestnut cultivars from Spain
}

\author{
S. Pereira-Lorenzo a,*, A.M. Ramos-Cabrer ${ }^{\text {a }}$, M.B. Díaz-Hernández ${ }^{\text {a }}$, \\ M. Ciordia-Ara ${ }^{\mathrm{b}}$, D. Ríos-Mesa ${ }^{\mathrm{c}}$ \\ ${ }^{a}$ Departamento de Producción Vexetal, Escola Politécnica Superior, Universidad de Santiago de Compostela, \\ Campus de Lugo 27002, Spain \\ ${ }^{\mathrm{b}}$ SERIDA (before Centro de Investigación Aplicada y Tecnología Agroalimentaria, CIATA), \\ Consejería de Medio Rural y Pesca, Principado de Asturias, 33300 Villaviciosa, Asturias, Spain \\ ${ }^{\mathrm{c}}$ Cabildo Insular de Tenerife, Área de Agricultura, Pza. España, 1, \\ 38001 Santa Cruz de Tenerife, Canary Islands, Spain
}

Received 9 July 2004; received in revised form 17 June 2005; accepted 24 August 2005

\begin{abstract}
Chestnut cultivation and production in Spain has employed grafted seedlings from selected local cultivars. Previously, we have characterised the Spanish cultivars by morphological and molecular markers. We are presenting in this paper the proximate analysis and mineral content for the main Spanish cultivars. A total of 131 samples were collected from 47 cultivars in six important Spanish chestnut production regions; located in the North such as Asturias, Castilla-León (El Bierzo) and Galicia; in the Central such as Extremadura and in the South such as Andalucía; as well as the Canary Islands, the southermost part of Spain near to North Africa. High variability in chemical composition between cultivars and regions corresponded to the high genetic variability between cultivars. Correlations with environmental parameters were low, indicating that differences found between regions were probably reflecting the differences between cultivars. In Central and Southern Spain, some cultivars presented lowest moisture content due to the low summer rainfall in these regions. Differences in starch and total sugar contents were high and were negatively correlated with each other. There was no negative correlation between nut size and total sugar content. Lowest values of fibre content and ease of digestibility were found in cultivars from Galicia and Extremadura. No significant differences in $\mathrm{Fe}, \mathrm{Zn}$ and $\mathrm{Cu}$ were found although $\mathrm{Zn}$ content is twice the value reported for European chestnuts. This work would be a valuable reference to chestnut quality for the food processing industry, nutritionists, breeders and growers alike.
\end{abstract}

(C) 2005 Elsevier B.V. All rights reserved.

Keywords: Castanea sativa; Starch; Total sugars; Fibre; Fat; Ashes; Proteins; Minerals

\section{Introduction}

Chestnut (Castanea sativa Mill.) is a multipurpose species that is cultivated for timber, nut, tannin, and contributes positively to the forestry landscape. It is distributed mainly in the Northern Hemisphere, in Asia mostly in China, Korea and Japan, in Southern Europe from Turkey to Atlantic Islands and in the United States (Pereira-Lorenzo and Ramos-Cabrer, 2004). Chestnut was only recently introduced into the Southern Hemisphere such as Chile, Argentina, Australia and New Zealand.

There are three main chestnut growing areas in the world: (i) Asia being the most important, mainly in China, where $C$. mollissima is found naturally as well as in cultivation; (ii) Southern Europe and Turkey is the second main area where $C$.

\footnotetext{
* Corresponding author. Tel.: +34 982252231; fax: +34 982285926.

E-mail address: spereira@lugo.usc.es (S. Pereira-Lorenzo).
}

sativa is predominant; (iii) in North-America, $C$. dentata was widespread naturally but is being substituted by hybrids with resistance to blight.

Chestnut has been cultivated for nut production in Northern Spain, mainly in Vasque Country, Asturias, Castilla-León (El Bierzo) and Galicia, in Central Spain in Extremadura and in Southern Spain in Andalucía (Fig. 1), and also some in Eastern Spain in Cataluña. Chestnut crops have been introduced into the Canary Islands, since colonization in the 16th century. In all these areas, growers selected the best trees for nut production by propagation via grafting over seedlings (Pereira-Lorenzo et al., 2001a,b).

Previous studies on chestnut composition was only on starch content comparing the three main species (McCarthy and Meredith, 1988), who obtained a higher starch content, $49 \%$ in C. dentata and C. mollissima in respect to $40 \%$ in C. sativa. Ferreira-Cardoso et al. (1993) compared the main Portuguese C. sativa cultivars starch content and found the lowest, $50 \%$ in 
Benfeita and the highest, 59\% in Lamela. Breisch (1995) explained the general values for the main chestnut nutrients and this work has formed the main basis for the current studies.

Although the selection of chestnut cultivars in Spain were made by nut quality such as big nut size, small number of polyembryonic nuts, and often for early harvesting (Ramos-Cabrer and Pereira-Lorenzo, in press), growers pointed out that some of the Spanish cultivars were sweeter or more easily digestible than others. We analysed the chestnut nutrients in order to assess if selection in respect to a scientific composition as a valuable resource for nutritionists, breeders and growers could be useful. This is the first comparative study of nutrient composition of Spanish chestnut cultivars from North to South and differentiating the main Spanish cultivars by their nutrient composition. It also provides a useful reference to the quality of each chestnut cultivar for the food processing industry, growers and breeders.

\section{Materials and methods}

\subsection{Plant material}

In this study, we have included 47 cultivars from six chestnut Spanish regions (Table 1; Fig. 1) (Pereira-Lorenzo et al., 2001a,b): 14 cultivars from Galicia, 9 from Asturias, 10 from Andalucía, 6 from Castilla-León (El Bierzo), 6 from Canary Islands (Tenerife) and 2 from Extremadura. These selected cultivars correspond to the most important cultivars in Spanish chestnut production. They were previously evaluated by morphology and isoenzymes to select the best cultivars in Galicia (Pereira-Lorenzo et al., 1996a,b; Ramos-Cabrer and Pereira-Lorenzo, in press). For statistical purpose, more than one tree was sampled for each cultivar, with a total of 131 samples analysed from these 47 cultivars.

\subsection{Samples}

One kilogram of chestnuts were collected in different regions of Spain at harvesting time (Pereira-Lorenzo et al., 2001a,b) and stored at $4{ }^{\circ} \mathrm{C}$. Within a week, nuts were hand peeled, weighed and dried in an oven at $80{ }^{\circ} \mathrm{C}$ until a constant weight was reached to determine the moisture content. All samples were ground to flour for further analysis in Laboratorio
Agrario e Fitopatolóxico de Galicia, Xunta de Galicia. Starch and total sugar content were assessed by a polarimeter using the Spanish Official Methodology (BOE, 2000). Neutral detergent fibre (NDF) and acid detergent fibre (ADF) were determined by the Van Soest detergent system (Van Soest et al., 1991) and crude fibre by AOAC (1990). For all fractions, fibres were determined using a Fibretec System (FOSS-Tecator). Fat content was estimated using a Soxtec System (FOSS-Tecator) (BOE, 1995). Crude protein was estimated as N-Kjeldahl applying 5.3 as a multiplicative factor (McCarthy and Meredith, 1988) using a digestor Gerhardt (BOE, 1995). Ash content was obtained by Spanish Official Methodology (BOE, 1995). Macro and microelements $\mathrm{Ca}, \mathrm{Mg}, \mathrm{Na}, \mathrm{K}, \mathrm{Cu}, \mathrm{Fe}, \mathrm{Mn}$ and $\mathrm{Zn}$ were determined by atomic absorption (AOAC, 1984) and $\mathrm{P}$ was estimated using a spectrophotometer UV-vis (BOE, 1995).

\subsection{Statistical analysis}

An analysis of variance (ANOVA) was conducted to estimate the effects of the region and the cultivar within region. The procedure PROC GLM of SAS for unbalanced data (SAS, 1988) was used according to the following model equation:

$$
X i(m) j=\mu+\mathrm{R} m+\mathrm{Ci}(m)+\varepsilon i(m) j
$$

where $X i(m) j$ is the observation of the Cultivar $i(i=1-14)$ within the Region $m(m=1-6)$ and the sample $j(j=1-7) ; \mu$ is the mean of all the observations; $\mathrm{Rm}, \mathrm{Ci}(m)$ and $\varepsilon i(m) j k$ are the effects of the Region $m$, the Accession $i$ within the Region $m$ and the error associated to the sample $k$ in the observation $i(m) j$, respectively. Student-Newman-Keuls multiple range test was used to indicate significant differences between means at the $5 \%$ level.

Pearson correlation coefficients were estimated for the environmental variables as annual average temperature, annual average rainfall, average rainfall during the summer period, and all the chemical components analysed in order to find possible relationships. In addition, Pearson correlation coefficients were estimated for the altitude and harvesting period of each sample with each chemical determination.

A principal components analysis (PCA) on the correlation matrix of the clone means of the nine main nutrients were carried out using the procedure PRINCOMP of SAS (SAS,

Table 1

Chestnut cultivars studied in the six regions from North to South of Spain

\begin{tabular}{|c|c|c|c|}
\hline Region & Cultivar (number of accessions per cultivar in parenthesis) & $\begin{array}{l}\text { Total } \\
\text { cultivars }\end{array}$ & $\begin{array}{l}\text { Number of } \\
\text { samples }\end{array}$ \\
\hline Andalucía & $\begin{array}{l}\text { Capilla (3), Comisaria (2), Dieguina (2), Helechal (2), Pilonga (4), } \\
\text { Planta Alajar (3), Rubia (3), Temprana (3), Tomasa (3), Vázquez (3) }\end{array}$ & 10 & 28 \\
\hline Asturias & $\begin{array}{l}\text { Chamberga (2), Doriga (2), Leinova (3), Llanisca (2), Mourisco (2), } \\
\text { Rapuga (2), Valduna (2), Verdeta (2), Zapatona (3) }\end{array}$ & 9 & 20 \\
\hline Canary Islands (Tenerife) & Castagrande (2), De Sala (2), Del Haya (2), Manso (2), Mulato (2), Redonda (2) & 6 & 12 \\
\hline Castilla-León (El Bierzo) & Injerta (2), Marela (2), Negral (6), Parede (2), Rapada (2), Verdello (3) & 6 & 17 \\
\hline Extremadura & Injerta (3), Verata (2) & 2 & 5 \\
\hline Galicia & $\begin{array}{l}\text { Amarelante (5), Famosa (5), Garrida (2), Inxerta (2), Longal (2), Loura (3), } \\
\text { Luguesa (4), Marela (2), Negral (4), Parede (7), Presa (3), Raigona (3), } \\
\text { Ventura (4), Verde (3) }\end{array}$ & 14 & 49 \\
\hline Spain & & 47 & 131 \\
\hline
\end{tabular}




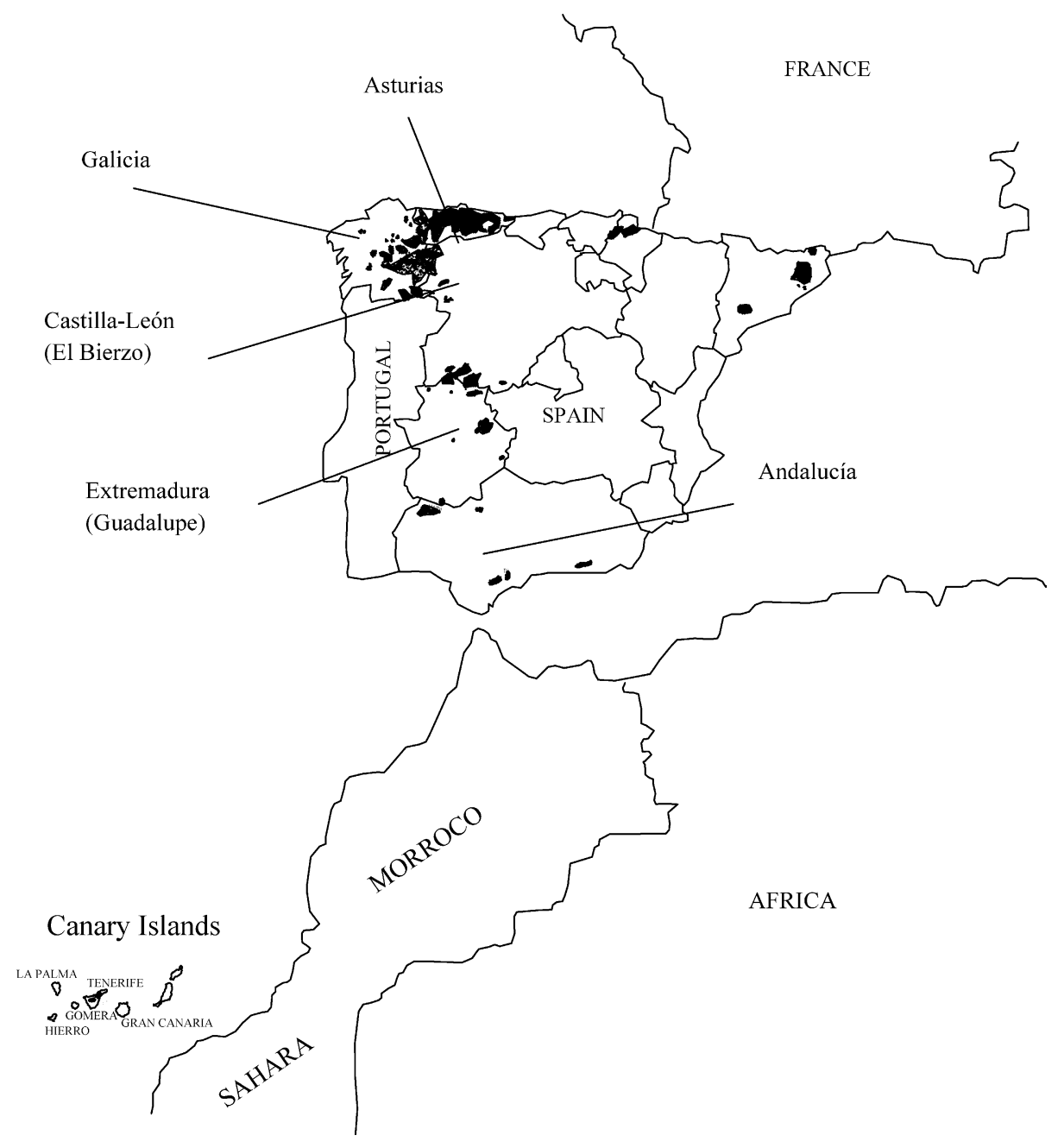

Fig. 1. Main area of Spanish chestnut crop (North to South).

1988) in order to show the variability between cultivars for those compounds.

\section{Results and discussion}

Some of the traits studied showed significant differences in both regions and cultivars (Tables 2-4). This corresponds with the high genetic diversity found between chestnut cultivars but also between regions due to the range of climatic conditions from Northern Spain to Canary Islands (Ramos-Cabrer and Pereira-Lorenzo, in press). Low Pearson correlations between environmental parameters, altitude and harvesting period and cultivars, demonstrated that the main differences between regions were due to the genetic differences found between the cultivars. The differences can be exploited for selection of cultivars for regional breeding purposes as well as for the growers targeting the market requirement.

\subsection{Proximate analysis}

Chestnuts have a very high moisture content, over $50 \%$ (Ensminger et al., 1995). High moisture content is essential for storage supply to the fresh market, but it also presents a mould problem during storage and delivery. The average moisture content of chestnuts in Spain was 54\%, close to the mean value referred to by McCarthy and Meredith (1988) and Breisch (1995) for European chestnuts; the range of which is between 49 and $60 \%$ for adequate conservation. These values were higher than American and Chinese chestnuts, with 44\% (McCarthy and Meredith, 1988). Significant regional differences were observed on average moisture content of nuts from South to North of the Iberian Peninsula. Andalucía (Southern Spain) and Extremadura (Central Spain) produced the nuts with lowest moisture content, whilst Canary Islands showed an intermediate situation, and Northern regions, such as Asturias, Castilla-León (El Bierzo) and Galicia showed the highest. The Pearson correlation between summer rainfall and moisture content was low but significant, $0.54(P<0.001)$. Some of the main cultivars of Central Andalucía; Pilonga, Temprana and Capilla, and Injerta from Extemadura, which produce big nuts, presented less than $49 \%$ in moisture content (Table 2).

Chestnuts store a reserve in the form of starch in cotyledons, and the content is three- to four-fold higher than found in other nuts (Ensminger et al., 1995). Average starch content for Spanish chestnuts was $57 \%$ (Table 2), higher than those obtained by McCarthy and Meredith (1988) who reported in 
Table 2

Average and $F$-values for moisture, starch and total sugar contents by cultivar and region from North to South of Spain

\begin{tabular}{|c|c|c|c|c|c|c|c|c|c|c|c|}
\hline \multirow{2}{*}{$\begin{array}{l}\text { Region } \\
\text { Andalucía }\end{array}$} & \multirow{2}{*}{$\begin{array}{l}\text { Cultivar } \\
\text { Capilla }\end{array}$} & \multirow{2}{*}{$\begin{array}{l}\text { Nuts/kga } \\
66\end{array}$} & \multirow{2}{*}{$\begin{array}{r}\text { No. } \\
2\end{array}$} & \multicolumn{2}{|c|}{$\begin{array}{l}\text { Moisture } \\
(\%)\end{array}$} & \multirow{2}{*}{$\begin{array}{r}\text { No. } \\
3\end{array}$} & \multicolumn{2}{|c|}{$\begin{array}{l}\text { Starch } \\
\text { (\% d.m.) }\end{array}$} & \multirow{2}{*}{$\begin{array}{l}N \\
3\end{array}$} & \multicolumn{2}{|c|}{$\begin{array}{l}\text { Total sugars } \\
\text { (\% d.m.) }\end{array}$} \\
\hline & & & & 47.5 & $a b^{\mathrm{b}}$ & & 51.5 & abcdef & & 17.1 & abcdef \\
\hline Andalucía & Comisaria & 88 & 2 & 56.0 & $a b$ & 2 & 59.8 & defgh & 2 & 10.7 & $\mathrm{ab}$ \\
\hline Andalucía & Dieguina & 87 & 2 & 58.2 & $\mathrm{~b}$ & 2 & 58.8 & defgh & 2 & 9.5 & $\mathrm{a}$ \\
\hline Andalucía & Helechal & 80 & 2 & 55.7 & $a b$ & 2 & 59.7 & defgh & 2 & 12.2 & $a b c$ \\
\hline Andalucía & Pilonga & 63 & 4 & 40.3 & $\mathrm{a}$ & 4 & 59.7 & defgh & 4 & 13.9 & abcde \\
\hline Andalucía & Planta Alajar & 71 & 2 & 54.2 & $a b$ & 3 & 53.8 & abcdefgh & 3 & 15.2 & abcdef \\
\hline Andalucía & Rubia & 49 & 2 & 51.3 & $a b$ & 3 & 56.2 & cdefgh & 3 & 13.8 & abcde \\
\hline Andalucía & Temprana & 61 & 3 & 45.3 & $a b$ & 3 & 55.8 & cdefgh & 3 & 17.1 & abcdef \\
\hline Andalucía & Tomasa & 44 & 3 & 53.7 & $a b$ & 3 & 53.2 & abcdefgh & 3 & 14.3 & abcde \\
\hline Andalucía & Vázquez & 73 & 2 & 53.6 & $a b$ & 3 & 58.6 & defgh & 3 & 12.7 & abcd \\
\hline Asturias & Chamberga & 91 & & & & 2 & 53.9 & abcdefgh & 2 & 17.1 & abcdef \\
\hline Asturias & Doriga & 135 & 2 & 55.6 & $a b$ & 2 & 57.1 & defgh & 2 & 15.7 & abcdef \\
\hline Asturias & Leinova & 142 & 2 & 55.9 & $a b$ & 3 & 55.0 & bcdefgh & 3 & 15.5 & abcdef \\
\hline Asturias & Llanisca & 125 & 2 & 54.9 & $a b$ & 2 & 59.4 & defgh & 2 & 12.1 & $a b c$ \\
\hline Asturias & Mourisco & 122 & 2 & 59.9 & $\mathrm{~b}$ & 2 & 53.5 & abcdefgh & 2 & 16.6 & abcdef \\
\hline Asturias & Rapuga & 135 & & & & 2 & 54.8 & bcdefgh & 2 & 17.0 & abcdef \\
\hline Asturias & Valduna & 91 & & & & 2 & 54.9 & bcdefgh & 2 & 17.0 & abcdef \\
\hline Asturias & Verdeta & 147 & 2 & 57.1 & $\mathrm{~b}$ & 2 & 56.9 & defgh & 2 & 13.4 & abcde \\
\hline Asturias & Zapatona & 97 & 2 & 60.1 & $\mathrm{~b}$ & 3 & 53.6 & abcdefgh & 3 & 15.8 & abcdef \\
\hline Bierzo & Injerta & 141 & & & & 2 & 49.8 & abcde & 2 & 21.9 & def \\
\hline Bierzo & Marela & 131 & & & & 2 & 59.0 & defgh & 2 & 14.6 & abcdef \\
\hline Bierzo & Negral & 79 & 6 & 56.9 & $\mathrm{~b}$ & 6 & 59.6 & defgh & 6 & 14.3 & abcde \\
\hline Bierzo & Parede & 115 & & & & 2 & 56.2 & cdefgh & 2 & 18.4 & abcdef \\
\hline Bierzo & Rapada & 146 & 2 & 58.5 & $\mathrm{~b}$ & 2 & 58.9 & defgh & 2 & 16.2 & abcdef \\
\hline Bierzo & Verdello & 112 & 2 & 53.4 & $a b$ & 3 & 59.3 & defgh & 3 & 16.9 & abcdef \\
\hline Canary Islands & Castagrande & 99 & 2 & 56.9 & $\mathrm{~b}$ & 2 & 43.2 & $a b$ & 2 & 20.7 & cdef \\
\hline Canary Islands & De Sala & 124 & 2 & 49.8 & $a b$ & 2 & 49.2 & abcd & 2 & 19.7 & bcdef \\
\hline Canary Islands & Del Haya & 155 & 2 & 50.8 & $a b$ & 2 & 43.9 & $a b c$ & 2 & 22.2 & ef \\
\hline Canary Islands & Manso & 130 & 2 & 52.7 & $a b$ & 2 & 52.5 & abcdef & 2 & 16.1 & abcdef \\
\hline Canary Islands & Mulato & 115 & 2 & 55.6 & $a b$ & 2 & 43.2 & $\mathrm{ab}$ & 2 & 22.2 & ef \\
\hline Canary Islands & Redonda & 103 & 2 & 50.8 & $a b$ & 2 & 42.2 & $\mathrm{a}$ & 2 & 23.4 & $\mathrm{f}$ \\
\hline Extremadura & Injerta & 78 & 2 & 48.7 & $a b$ & 3 & 55.8 & cdefgh & 3 & 15.0 & abcdef \\
\hline Extremadura & Verata & 90 & 2 & 52.7 & $a b$ & 2 & 60.5 & defgh & 2 & 12.1 & $a b c$ \\
\hline Galicia & Amarelante & 82 & 4 & 53.4 & $a b$ & 5 & 62.1 & defgh & 5 & 12.3 & $a b c$ \\
\hline Galicia & Famosa & 86 & & & & 5 & 57.6 & defgh & 5 & 16.2 & abcdef \\
\hline Galicia & Garrida & 85 & 2 & 56.2 & $a b$ & 2 & 62.2 & defgh & 2 & 11.8 & $a b c$ \\
\hline Galicia & Inxerta & 81 & 2 & 53.7 & $a b$ & 2 & 64.6 & fgh & 2 & 10.1 & $\mathrm{a}$ \\
\hline Galicia & Longal & 118 & & & & 2 & 66.5 & gh & 2 & 10.3 & $a b$ \\
\hline Galicia & Loura & 86 & 3 & 58.7 & $\mathrm{~b}$ & 3 & 60.0 & defgh & 3 & 11.2 & $\mathrm{ab}$ \\
\hline Galicia & Luguesa & 83 & 4 & 57.7 & $\mathrm{~b}$ & 4 & 53.9 & abcdefgh & 4 & 15.5 & abcdef \\
\hline Galicia & Marela & 126 & 2 & 57.7 & $\mathrm{~b}$ & 2 & 59.7 & defgh & 2 & 14.0 & abcde \\
\hline Galicia & Negral & 118 & 4 & 50.4 & $\mathrm{ab}$ & 4 & 57.4 & defgh & 4 & 16.1 & abcdef \\
\hline Galicia & Parede & 135 & 7 & 52.9 & $a b$ & 7 & 59.7 & defgh & 7 & 14.7 & abcdef \\
\hline Galicia & Presa & 95 & 2 & 55.4 & $a b$ & 3 & 58.1 & defgh & 3 & 13.9 & abcde \\
\hline Galicia & Raigona & 128 & 3 & 57.7 & $\mathrm{~b}$ & 3 & 63.4 & efgh & 3 & 11.6 & $a b c$ \\
\hline Galicia & Ventura & 87 & 3 & 58.5 & $\mathrm{~b}$ & 4 & 57.9 & defgh & 4 & 14.6 & abcdef \\
\hline Galicia & Verde & 105 & 3 & 58.0 & $\mathrm{~b}$ & 3 & 57.5 & defgh & 3 & 14.1 & abcde \\
\hline$F$-values: & & & & & & 131 & & & & & \\
\hline Region & & & & 3.6 & $* *$ & & 4164.2 & $* * * *$ & & 13.8 & $* * * *$ \\
\hline Cultivar (Region) & & & & 1.4 & ns & & 1.8 & $*$ & & 1.1 & $\mathrm{~ns}$ \\
\hline Andalucía & & & 24 & 50.5 & $\mathrm{a}$ & 28 & 56.5 & $\mathrm{~b}$ & 28 & 14.0 & $\mathrm{a}$ \\
\hline Asturias & & & 19 & 56.2 & $\mathrm{~b}$ & 20 & 56.0 & $\mathrm{~b}$ & 20 & 15.5 & $\mathrm{a}$ \\
\hline Bierzo & & & 12 & 55.6 & $\mathrm{~b}$ & 17 & 57.9 & $\mathrm{~b}$ & 17 & 16.3 & $\mathrm{a}$ \\
\hline Canary Islands & & & 12 & 52.8 & $a b$ & 12 & 45.7 & $\mathrm{a}$ & 12 & 20.7 & $\mathrm{~b}$ \\
\hline Extremadura & & & 4 & 50.7 & $\mathrm{a}$ & 5 & 57.6 & $\mathrm{~b}$ & 5 & 13.8 & $\mathrm{a}$ \\
\hline Galicia & & & 38 & 56.1 & $\mathrm{~b}$ & 46 & 59.5 & $\mathrm{~b}$ & 46 & 13.7 & $\mathrm{a}$ \\
\hline Spain & & & 109 & 54.3 & & 131 & 56.7 & & 131 & 15.1 & \\
\hline
\end{tabular}

ns: non significant.

${ }^{a}$ Nuts/kg (Pereira-Lorenzo et al., 2001c; Pereira-Lorenzo et al., 2005; Pereira-Lorenzo and Ramos-Cabrer, 2003; Ramos-Cabrer et al., 2003).

${ }^{\mathrm{b}}$ For each column, means with different letters are significantly different $(P=0.05)$ according to Student-Newman-Keuls multiple range test.

* Significant at $P \leq 0.05$, respectively.

** Significant at $P \leq 0.01$, respectively.

***** Significant at $P \leq 0.0001$, respectively. 
Table 3

Average and $F$-values for acid detergent fibre, neutral detergent fibre, crude fibre, fats, crude proteins, ashes in percentage of dried matter

\begin{tabular}{|c|c|c|c|c|c|c|c|c|c|c|c|c|c|c|c|c|c|c|c|}
\hline Region & Cultivar & No. & $\mathrm{ADF}$ & & No. & $\mathrm{NDF}$ & & No. & $\begin{array}{l}\text { Crude } \\
\text { fibre }\end{array}$ & & No. & Fats & & No. & $\begin{array}{l}\text { Crude } \\
\text { proteins }\end{array}$ & & No. & Ashes & \\
\hline Andalucía & Capilla & 2 & 4.5 & $a^{a}$ & 2 & 9.4 & $\mathrm{a}$ & 2 & 3.7 & a & 2 & 3.6 & bcd & 2 & 5.3 & $\mathrm{a}$ & 2 & 2.7 & $a b c$ \\
\hline Andalucía & Comisaria & 2 & 3.1 & $\mathrm{a}$ & 2 & 15.3 & $a b$ & 2 & 2.7 & a & 2 & 2.1 & $a b c$ & 2 & 5.7 & $\mathrm{a}$ & 2 & 2.5 & $a b c$ \\
\hline Andalucía & Dieguina & 2 & 2.6 & $\mathrm{a}$ & 2 & 15.6 & $\mathrm{ab}$ & 2 & 2.4 & $\mathrm{a}$ & 2 & 1.9 & $\mathrm{ab}$ & 2 & 9.6 & $\mathrm{~b}$ & 2 & 3.2 & $\mathrm{c}$ \\
\hline Andalucía & Helechal & 2 & 2.6 & $\mathrm{a}$ & 2 & 13.7 & $a b$ & 2 & 2.6 & $\mathrm{a}$ & 2 & 2.5 & abcd & 2 & 4.9 & $\mathrm{a}$ & 2 & 2.2 & $a b$ \\
\hline Andalucía & Pilonga & 4 & 3.9 & $\mathrm{a}$ & 4 & 28.5 & $\mathrm{~b}$ & 3 & 3.0 & $\mathrm{a}$ & 4 & 4.0 & $\mathrm{~d}$ & 4 & 5.2 & $\mathrm{a}$ & 4 & 2.7 & $a b c$ \\
\hline Andalucía & Planta Alajar & 2 & 3.1 & $\mathrm{a}$ & 2 & 14.7 & $a b$ & 2 & 2.3 & $\mathrm{a}$ & 2 & 2.3 & abcd & 2 & 6.9 & $\mathrm{a}$ & 2 & 2.1 & $\mathrm{ab}$ \\
\hline Andalucía & Rubia & 2 & 4.0 & $\mathrm{a}$ & 2 & 14.3 & $\mathrm{ab}$ & 2 & 2.6 & $\mathrm{a}$ & 2 & 2.9 & abcd & 2 & 5.6 & $\mathrm{a}$ & 2 & 2.2 & $a b$ \\
\hline Andalucía & Temprana & 3 & 4.2 & $\mathrm{a}$ & 3 & 23.2 & $a b$ & 2 & 3.5 & $\mathrm{a}$ & 3 & 3.9 & $\mathrm{~cd}$ & 3 & 5.4 & $\mathrm{a}$ & 3 & 2.1 & $a b$ \\
\hline Andalucía & Tomasa & 2 & 4.3 & $\mathrm{a}$ & 2 & 21.0 & $a b$ & 2 & 2.7 & $\mathrm{a}$ & 2 & 3.4 & abcd & 2 & 6.4 & $\mathrm{a}$ & 2 & 2.3 & $a b c$ \\
\hline Andalucía & Vazquez & 2 & 2.4 & $\mathrm{a}$ & 2 & 15.6 & $a b$ & 2 & 3.2 & $\mathrm{a}$ & 2 & 2.2 & abcd & 2 & 6.3 & $\mathrm{a}$ & 2 & 2.2 & $a b c$ \\
\hline Asturias & Chamberga & 2 & 3.1 & $\mathrm{a}$ & 2 & 17.5 & $a b$ & 2 & 2.9 & $\mathrm{a}$ & 2 & 3.3 & abcd & 2 & 4.5 & $\mathrm{a}$ & 2 & 2.3 & $a b c$ \\
\hline Asturias & Doriga & 2 & 2.5 & $\mathrm{a}$ & 2 & 13.5 & $a b$ & 2 & 2.5 & $\mathrm{a}$ & 2 & 3.5 & abcd & 2 & 5.9 & $\mathrm{a}$ & 2 & 2.3 & $a b c$ \\
\hline Asturias & Leinova & 2 & 3.0 & $\mathrm{a}$ & 2 & 17.7 & $a b$ & 2 & 2.3 & $\mathrm{a}$ & 2 & 3.1 & abcd & 2 & 6.2 & $\mathrm{a}$ & 2 & 2.0 & $a b$ \\
\hline Asturias & Llanisca & 2 & 2.8 & $\mathrm{a}$ & 2 & 16.4 & $a b$ & 2 & 2.3 & $\mathrm{a}$ & 2 & 1.7 & $\mathrm{a}$ & 2 & 5.2 & $\mathrm{a}$ & 2 & 2.2 & $a b c$ \\
\hline Asturias & Rapuga & 2 & 3.0 & $\mathrm{a}$ & 2 & 15.9 & $a b$ & 2 & 2.2 & $\mathrm{a}$ & 2 & 3.3 & abcd & 2 & 5.6 & $\mathrm{a}$ & 2 & 2.2 & $a b$ \\
\hline Asturias & Valduna & 2 & 3.5 & $\mathrm{a}$ & 2 & 16.4 & $a b$ & 2 & 2.7 & $\mathrm{a}$ & 2 & 3.8 & bcd & 2 & 4.8 & $\mathrm{a}$ & 2 & 2.2 & $a b c$ \\
\hline Asturias & Verdeta & 2 & 3.1 & $\mathrm{a}$ & 2 & 17.1 & $a b$ & 2 & 3.0 & $\mathrm{a}$ & 2 & 2.8 & abcd & 2 & 6.4 & $\mathrm{a}$ & 2 & 2.2 & $a b$ \\
\hline Asturias & Zapatona & 2 & 4.3 & $\mathrm{a}$ & 2 & 18.3 & $a b$ & 2 & 3.4 & $\mathrm{a}$ & 2 & 2.3 & abcd & 2 & 6.9 & $\mathrm{a}$ & 2 & 2.9 & $\mathrm{bc}$ \\
\hline Bierzo & Injerta & 2 & 3.6 & $\mathrm{a}$ & 2 & 15.1 & $a b$ & 2 & 2.7 & $\mathrm{a}$ & 2 & 2.8 & abcd & 2 & 7.3 & $\mathrm{a}$ & 2 & 2.7 & $a b c$ \\
\hline Bierzo & Marela & 2 & 2.6 & $\mathrm{a}$ & 2 & 15.0 & $a b$ & 2 & 2.4 & $\mathrm{a}$ & 2 & 2.7 & abcd & 2 & 6.5 & $\mathrm{a}$ & 2 & 2.2 & $a b c$ \\
\hline Bierzo & Negral & 5 & 3.0 & $\mathrm{a}$ & 5 & 18.3 & $a b$ & 5 & 3.1 & $\mathrm{a}$ & 5 & 2.6 & abcd & 5 & 6.6 & $\mathrm{a}$ & 5 & 2.3 & $a b c$ \\
\hline Bierzo & Parede & 2 & 2.9 & $\mathrm{a}$ & 2 & 18.2 & $a b$ & 2 & 2.3 & $\mathrm{a}$ & 2 & 3.9 & $\mathrm{~cd}$ & 2 & 6.0 & $\mathrm{a}$ & 2 & 2.2 & $a b$ \\
\hline Bierzo & Rapada & 2 & 2.8 & $\mathrm{a}$ & 2 & 16.8 & $a b$ & 2 & 2.6 & $\mathrm{a}$ & 2 & 2.8 & abcd & 2 & 5.9 & $\mathrm{a}$ & 2 & 2.5 & $a b c$ \\
\hline Bierzo & Verdello & 3 & 3.2 & $\mathrm{a}$ & 3 & 20.0 & $\mathrm{ab}$ & 3 & 2.6 & a & 3 & 3.6 & abcd & 3 & 5.2 & a & 3 & 2.0 & $a b$ \\
\hline Extremadura & Injerta & 2 & 2.8 & $\mathrm{a}$ & 2 & 18.3 & $a b$ & 2 & 2.3 & $\mathrm{a}$ & 2 & 2.7 & abcd & 2 & 5.3 & $\mathrm{a}$ & 2 & 1.8 & $\mathrm{a}$ \\
\hline Extremadura & Verata & 2 & 2.7 & $\mathrm{a}$ & 2 & 14.1 & $a b$ & 2 & 2.7 & $\mathrm{a}$ & 2 & 2.9 & abcd & 2 & 6.1 & $\mathrm{a}$ & 2 & 2.0 & $a b$ \\
\hline Galicia & Amarelante & 4 & 2.5 & $\mathrm{a}$ & 4 & 18.5 & $a b$ & 4 & 2.4 & $\mathrm{a}$ & 4 & 3.4 & abcd & 4 & 5.0 & $\mathrm{a}$ & 4 & 2.1 & $a b$ \\
\hline Galicia & Famosa & 4 & 3.0 & $\mathrm{a}$ & 4 & 17.0 & $a b$ & 4 & 2.6 & a & 4 & 2.3 & abcd & 4 & 5.3 & a & 4 & 2.4 & $a b c$ \\
\hline Galicia & Garrida & 2 & 2.7 & $\mathrm{a}$ & 2 & 15.5 & $a b$ & 2 & 2.6 & a & 2 & 2.8 & abcd & 2 & 7.2 & $\mathrm{a}$ & 2 & 2.5 & $a b c$ \\
\hline Galicia & Inxerta & 2 & 2.5 & $\mathrm{a}$ & 2 & 17.8 & $a b$ & 2 & 2.5 & $\mathrm{a}$ & 2 & 3.4 & abcd & 2 & 5.3 & $\mathrm{a}$ & 2 & 2.2 & $a b c$ \\
\hline Galicia & Longal & 2 & 2.3 & $\mathrm{a}$ & 2 & 12.1 & $\mathrm{a}$ & 2 & 2.3 & a & 2 & 2.4 & abcd & 2 & 5.2 & a & 2 & 2.5 & $a b c$ \\
\hline Galicia & Loura & 3 & 2.7 & $\mathrm{a}$ & 3 & 14.6 & $a b$ & 3 & 2.5 & $\mathrm{a}$ & 3 & 3.4 & abcd & 3 & 7.4 & $\mathrm{a}$ & 3 & 2.6 & $a b c$ \\
\hline Galicia & Luguesa & 2 & 3.2 & $\mathrm{a}$ & 2 & 18.1 & $a b$ & 2 & 2.5 & $\mathrm{a}$ & 2 & 3.0 & abcd & 2 & 5.6 & $\mathrm{a}$ & 2 & 2.1 & $a b$ \\
\hline Galicia & Marela & 2 & 2.5 & a & 2 & 14.9 & $\mathrm{ab}$ & 2 & 2.2 & a & 2 & 3.7 & bcd & 2 & 5.0 & $\mathrm{a}$ & 2 & 2.2 & $a b c$ \\
\hline Galicia & Negral & 4 & 3.0 & $\mathrm{a}$ & 4 & 15.8 & $a b$ & 4 & 2.9 & $\mathrm{a}$ & 4 & 2.8 & abcd & 4 & 6.3 & $\mathrm{a}$ & 4 & 2.2 & $a b c$ \\
\hline Galicia & Parede & 7 & 2.3 & $\mathrm{a}$ & 7 & 15.5 & $a b$ & 7 & 2.3 & $\mathrm{a}$ & 7 & 4.0 & d & 7 & 5.1 & $\mathrm{a}$ & 7 & 2.0 & $a b$ \\
\hline Galicia & Presa & 2 & 3.5 & a & 2 & 23.2 & $a b$ & 2 & 3.5 & $\mathrm{a}$ & 2 & 3.5 & abcd & 2 & 5.2 & $\mathrm{a}$ & 2 & 2.1 & $a b$ \\
\hline Galicia & Raigona & 3 & 3.3 & a & 3 & 22.3 & $a b$ & 3 & 3.2 & a & 3 & 2.6 & abcd & 3 & 5.6 & a & 3 & 2.4 & $a b c$ \\
\hline Galicia & Ventura & 3 & 2.7 & $\mathrm{a}$ & 3 & 14.4 & $a b$ & 3 & 2.6 & $\mathrm{a}$ & 3 & 2.7 & abcd & 3 & 5.6 & $\mathrm{a}$ & 3 & 2.2 & $a b c$ \\
\hline Galicia & Verde & 3 & 2.7 & a & 3 & 17.6 & $a b$ & 3 & 2.5 & $\mathrm{a}$ & 3 & 3.0 & abcd & 3 & 6.6 & $\mathrm{a}$ & 3 & 2.4 & $a b c$ \\
\hline Region & & & 4.1 & $* *$ & & 0.2 & ns & & 0.9 & ns & & 0.6 & $\mathrm{~ns}$ & & 1.4 & ns & & 3.4 & $*$ \\
\hline Cultivar (Region) & & & 1.7 & * & & 2.0 & $* *$ & & 1.0 & $\mathrm{~ns}$ & & 3.9 & $* * * * *$ & & 2.0 & $* *$ & & 2.3 & $* *$ \\
\hline \multicolumn{20}{|l|}{ Region } \\
\hline Andalucía & & 23 & 3.5 & $\mathrm{~b}$ & 23 & 18.4 & $\mathrm{a}$ & 21 & 2.9 & $\mathrm{a}$ & 23 & 3.0 & $\mathrm{a}$ & 23 & 6.0 & $\mathrm{a}$ & 23 & 2.4 & $\mathrm{~b}$ \\
\hline Asturias & & 21 & 3.0 & $\mathrm{ab}$ & 20 & 16.2 & $\mathrm{a}$ & 20 & 2.6 & $\mathrm{a}$ & 20 & 3.2 & $\mathrm{a}$ & 20 & 5.5 & $\mathrm{a}$ & 20 & 2.2 & $\mathrm{~b}$ \\
\hline Bierzo & & 15 & 3.0 & $\mathrm{ab}$ & 15 & 17.7 & $\mathrm{a}$ & 15 & 2.7 & $\mathrm{a}$ & 15 & 3.0 & $\mathrm{a}$ & 15 & 6.3 & $\mathrm{a}$ & 15 & 2.3 & $\mathrm{~b}$ \\
\hline \multicolumn{20}{|l|}{ Canary Islands } \\
\hline Extremadura & & 4 & 2.7 & $\mathrm{a}$ & 4 & 16.2 & $\mathrm{a}$ & 4 & 2.5 & $\mathrm{a}$ & 4 & 2.8 & $\mathrm{a}$ & 4 & 5.7 & $\mathrm{a}$ & 4 & 1.9 & $\mathrm{a}$ \\
\hline Galicia & & 40 & 2.8 & $\mathrm{a}$ & 40 & 17.0 & $\mathrm{a}$ & 40 & 2.6 & $\mathrm{a}$ & 40 & 3.0 & $\mathrm{a}$ & 40 & 5.8 & $\mathrm{a}$ & 40 & 2.3 & $\mathrm{~b}$ \\
\hline Spain & & 103 & 3.0 & & 102 & 17.2 & & 100 & 2.7 & & 102 & 3.0 & & 102 & 5.8 & & 102 & 2.3 & \\
\hline
\end{tabular}

ns: non significant.

${ }^{a}$ For each column, means with different letters are significantly different $(P=0.05)$ according to Student-Newman-Keuls multiple range test.

* Significant at $P \leq 0.05$, respectively.

**** Significant at $P \leq 0.01$, respectively.

******* Significant at $P \leq 0.0001$, respectively. 
Table 4

Mineral content of the most important Spanish cultivars

\begin{tabular}{|c|c|c|c|c|c|c|c|c|c|c|c|c|c|c|c|c|c|c|c|c|c|c|c|c|c|c|c|c|}
\hline Region & Cultivar & No. & $\mathrm{K}(\%)$ & & No. & $\mathrm{P}(\%)$ & & No. & $\mathrm{Ca}(\%)$ & & No. & $\operatorname{Mg}(\%)$ & & No. & $\mathrm{Na}(\%)$ & & No. & $\begin{array}{l}\mathrm{Zn} \\
(\mathrm{ppm})\end{array}$ & & No. & $\begin{array}{l}\mathrm{Cu} \\
(\mathrm{ppm})\end{array}$ & & No. & $\mathrm{Fe}(\mathrm{ppm})$ & & No. & $\begin{array}{l}\mathrm{Mn} \\
(\mathrm{ppm})\end{array}$ & \\
\hline Andalucía & Comisaria & 2 & 0.945 & $\mathrm{a}^{\mathrm{a}}$ & 2 & 0.167 & $a b$ & 2 & 0.045 & $a b$ & 2 & 0.084 & $\mathrm{~cd}$ & 2 & 0.004 & a & 2 & 12.9 & $\mathrm{a}$ & 2 & 9.0 & $\mathrm{a}$ & 2 & 17.0 & $\mathrm{a}$ & 2 & 82.9 & $a b c$ \\
\hline Andalucía & Dieguina & 2 & 1.130 & $\mathrm{a}$ & 2 & 0.199 & $a b$ & 2 & 0.034 & $a b$ & 2 & 0.100 & $\mathrm{~d}$ & & - & & 2 & 12.9 & $\mathrm{a}$ & 2 & 7.7 & $\mathrm{a}$ & 2 & 16.1 & $\mathrm{a}$ & 2 & 51.5 & $a b$ \\
\hline Andalucía & Helechal & 2 & 0.815 & $\mathrm{a}$ & 2 & 0.165 & $a b$ & 2 & 0.037 & $a b$ & 2 & 0.078 & $\mathrm{bc}$ & 2 & 0.003 & $\mathrm{a}$ & 2 & 11.8 & $\mathrm{a}$ & 2 & 6.4 & $\mathrm{a}$ & 2 & 13.5 & $\mathrm{a}$ & 2 & 62.7 & $a b$ \\
\hline Andalucía & Pilonga & 4 & 0.923 & $\mathrm{a}$ & 4 & 0.113 & $a b$ & 4 & 0.051 & $a b$ & 4 & 0.070 & $a b c$ & 4 & 0.010 & a & 4 & 10.8 & a & 4 & 6.5 & a & 4 & 17.5 & $\mathrm{a}$ & 4 & 29.5 & $a b$ \\
\hline Andalucía & Temprana & 3 & 0.870 & a & 3 & 0.167 & $a b$ & 3 & 0.052 & $a b$ & 3 & 0.064 & $a b c$ & 3 & 0.010 & a & 3 & 12.0 & a & 3 & 6.3 & a & 3 & 18.7 & $\mathrm{a}$ & 3 & 18.0 & $\mathrm{a}$ \\
\hline Asturias & Doriga & 2 & 0.885 & $\mathrm{a}$ & 2 & 0.130 & $a b$ & 2 & 0.030 & $a b$ & 2 & 0.064 & $a b c$ & 2 & 0.003 & a & 2 & 13.5 & a & 2 & 10.3 & a & 2 & 23.8 & $\mathrm{a}$ & 2 & 33.9 & $a b$ \\
\hline Asturias & Llanisca & 2 & 0.835 & $\mathrm{a}$ & 2 & 0.145 & $a b$ & 2 & 0.045 & $\mathrm{ab}$ & 2 & 0.056 & $a b c$ & & - & & 2 & 12.7 & a & 2 & 6.3 & a & 2 & 15.4 & $\mathrm{a}$ & 2 & 124.6 & $\mathrm{c}$ \\
\hline Asturias & Verdeta & 2 & 0.855 & $\mathrm{a}$ & 2 & 0.140 & $a b$ & 2 & 0.034 & $a b$ & 2 & 0.059 & $a b c$ & & - & & 2 & 10.4 & $\mathrm{a}$ & 2 & 6.3 & $\mathrm{a}$ & 2 & 16.4 & $\mathrm{a}$ & 2 & 34.4 & $a b$ \\
\hline Bierzo & Marela & 2 & 0.945 & $\mathrm{a}$ & 2 & 0.300 & $\mathrm{~b}$ & 2 & 0.034 & $a b$ & 2 & 0.055 & $a b$ & 2 & 0.005 & $\mathrm{a}$ & 2 & 11.5 & $\mathrm{a}$ & 2 & 7.0 & $\mathrm{a}$ & 2 & 17.5 & $\mathrm{a}$ & 2 & 38.5 & $a b$ \\
\hline Bierzo & Negral & 5 & 0.986 & $\mathrm{a}$ & 5 & 0.270 & $a b$ & 5 & 0.028 & $\mathrm{a}$ & 5 & 0.080 & $\mathrm{bc}$ & 5 & 0.003 & $\mathrm{a}$ & 5 & 14.2 & $\mathrm{a}$ & 5 & 7.0 & $\mathrm{a}$ & 5 & 19.0 & $\mathrm{a}$ & 5 & 32.4 & $a b$ \\
\hline Bierzo & Rapada & 2 & 1.040 & $\mathrm{a}$ & 2 & 0.295 & $\mathrm{~b}$ & 2 & 0.058 & $\mathrm{bc}$ & 2 & 0.074 & $a b c$ & 2 & 0.007 & $\mathrm{a}$ & 2 & 12.5 & $\mathrm{a}$ & 2 & 7.5 & $\mathrm{a}$ & 2 & 21.0 & $\mathrm{a}$ & 2 & 17.0 & $\mathrm{a}$ \\
\hline Bierzo & Verdello & 2 & 0.810 & $\mathrm{a}$ & 2 & 0.245 & $a b$ & 2 & 0.036 & $\mathrm{ab}$ & 2 & 0.054 & $\mathrm{ab}$ & 2 & 0.006 & $\mathrm{a}$ & 2 & 10.0 & $\mathrm{a}$ & 2 & 6.0 & $\mathrm{a}$ & 2 & 17.5 & a & 2 & 41.5 & $a b$ \\
\hline Extremadura & Verata & 2 & 0.800 & $\mathrm{a}$ & 2 & 0.110 & $a b$ & 2 & 0.035 & $a b$ & 2 & 0.067 & $a b c$ & & - & & 2 & 12.0 & $\mathrm{a}$ & 2 & 8.1 & $\mathrm{a}$ & 2 & 15.5 & $\mathrm{a}$ & 2 & 23.0 & $\mathrm{a}$ \\
\hline Galicia & Amarelante & 4 & 0.853 & $\mathrm{a}$ & 4 & 0.196 & $a b$ & 4 & 0.035 & $a b$ & 4 & 0.058 & $a b c$ & 4 & 0.026 & $\mathrm{a}$ & 4 & 12.0 & $\mathrm{a}$ & 4 & 6.0 & $\mathrm{a}$ & 4 & 17.3 & $\mathrm{a}$ & 4 & 17.8 & $\mathrm{a}$ \\
\hline Galicia & Famosa & 2 & 0.965 & $\mathrm{a}$ & 2 & 0.141 & $a b$ & 2 & 0.044 & $a b$ & 2 & 0.066 & $a b c$ & 2 & 0.024 & $\mathrm{a}$ & 2 & 13.0 & $\mathrm{a}$ & 2 & 9.0 & $\mathrm{a}$ & 2 & 21.0 & $\mathrm{a}$ & 2 & 92.5 & $\mathrm{bc}$ \\
\hline Galicia & Garrida & 2 & 0.930 & $\mathrm{a}$ & 2 & 0.137 & $a b$ & 2 & 0.044 & $a b$ & 2 & 0.063 & $a b c$ & 2 & 0.005 & $\mathrm{a}$ & 2 & 12.0 & $\mathrm{a}$ & 2 & 5.5 & $\mathrm{a}$ & 2 & 18.5 & $\mathrm{a}$ & 2 & 19.0 & $\mathrm{a}$ \\
\hline Galicia & Inxerta & 2 & 0.935 & $\mathrm{a}$ & 2 & 0.305 & $\mathrm{~b}$ & 2 & 0.048 & $a b$ & 2 & 0.064 & $a b c$ & 2 & 0.008 & $\mathrm{a}$ & 2 & 11.5 & $\mathrm{a}$ & 2 & 7.5 & $\mathrm{a}$ & 2 & 14.0 & $\mathrm{a}$ & 2 & 52.0 & $a b$ \\
\hline Galicia & Longal & 2 & 0.915 & $\mathrm{a}$ & 2 & 0.068 & $\mathrm{a}$ & 2 & 0.026 & $\mathrm{a}$ & 2 & 0.049 & $\mathrm{a}$ & 2 & 0.005 & a & 2 & 13.5 & a & 2 & 8.5 & a & 2 & 23.0 & $\mathrm{a}$ & 2 & 56.5 & $a b$ \\
\hline Galicia & Loura & 3 & 0.917 & $\mathrm{a}$ & 3 & 0.151 & $a b$ & 3 & 0.045 & $a b$ & 3 & 0.068 & $a b c$ & & - & & 3 & 12.9 & a & 3 & 7.2 & a & 3 & 18.8 & $\mathrm{a}$ & 3 & 27.1 & $a b$ \\
\hline Galicia & Marela & 2 & 0.865 & a & 2 & 0.135 & $a b$ & 2 & 0.043 & $a b$ & 2 & 0.084 & $\mathrm{~cd}$ & & - & & 2 & 10.5 & $\mathrm{a}$ & 2 & 8.4 & $\mathrm{a}$ & 2 & 15.1 & $\mathrm{a}$ & 2 & 50.2 & $a b$ \\
\hline Galicia & Negral & 4 & 0.910 & $\mathrm{a}$ & 4 & 0.253 & $a b$ & 4 & 0.039 & $a b$ & 4 & 0.074 & $a b c$ & 4 & 0.003 & $\mathrm{a}$ & 4 & 11.8 & $\mathrm{a}$ & 4 & 6.3 & $\mathrm{a}$ & 4 & 18.8 & $\mathrm{a}$ & 4 & 18.8 & $\mathrm{a}$ \\
\hline Galicia & Parede & 7 & 0.789 & $\mathrm{a}$ & 7 & 0.202 & $a b$ & 7 & 0.048 & $a b$ & 7 & 0.069 & $a b c$ & 7 & 0.006 & $\mathrm{a}$ & 7 & 10.4 & $\mathrm{a}$ & 7 & 7.4 & $\mathrm{a}$ & 7 & 17.5 & $\mathrm{a}$ & 7 & 49.5 & $a b$ \\
\hline Galicia & Presa & 2 & 0.815 & a & 2 & 0.137 & $a b$ & 2 & 0.037 & $a b$ & 2 & 0.063 & $a b c$ & 2 & 0.004 & $\mathrm{a}$ & 2 & 11.0 & $\mathrm{a}$ & 2 & 6.0 & $\mathrm{a}$ & 2 & 15.0 & $\mathrm{a}$ & 2 & 32.0 & $a b$ \\
\hline Galicia & Raigona & 3 & 1.030 & $\mathrm{a}$ & 3 & 0.243 & $a b$ & 3 & 0.037 & $a b$ & 3 & 0.077 & $a b c$ & 3 & 0.017 & $\mathrm{a}$ & 3 & 12.3 & $\mathrm{a}$ & 3 & 7.0 & $\mathrm{a}$ & 3 & 18.7 & $\mathrm{a}$ & 3 & 18.3 & $\mathrm{a}$ \\
\hline Galicia & Ventura & 3 & 0.853 & $\mathrm{a}$ & 3 & 0.173 & $a b$ & 3 & 0.042 & $a b$ & 3 & 0.057 & $a b c$ & 3 & 0.005 & $\mathrm{a}$ & 3 & 13.0 & $\mathrm{a}$ & 3 & 6.7 & $\mathrm{a}$ & 3 & 21.0 & $\mathrm{a}$ & 3 & 32.0 & $a b$ \\
\hline Galicia & Verde & 3 & 0.917 & $\mathrm{a}$ & 3 & 0.167 & $a b$ & 3 & 0.072 & c & 3 & 0.064 & $a b c$ & 2 & 0.006 & $\mathrm{a}$ & 3 & 19.0 & $\mathrm{a}$ & 3 & 7.1 & a & 3 & 20.9 & a & 3 & 20.7 & $\mathrm{a}$ \\
\hline Region & & & 3.243 & * & & 7.499 & ******* & & 1.180 & ns & & 8.734 & $* * * * *$ & & 0.387 & ns & & 0.5 & ns & & 0.5 & ns & & 0.5 & $\mathrm{~ns}$ & & 3.4 & * \\
\hline Cultivar (region) & & & 1.621 & ns & & 1.821 & * & & 4.185 & $* * * *$ & & 4.329 & $* * * * *$ & & 0.663 & ns & & 1.1 & ns & & 1.0 & ns & & 1.0 & ns & & 3.5 & **** \\
\hline Andalucía & & 13 & 0.929 & $a b$ & 13 & 0.155 & a & 13 & 0.045 & $\mathrm{a}$ & 13 & 0.077 & $\mathrm{~b}$ & 11 & 0.008 & $\mathrm{a}$ & 13 & 11.8 & $\mathrm{a}$ & 13 & 7.0 & $\mathrm{a}$ & 13 & 16.9 & $\mathrm{a}$ & 13 & 43.6 & $\mathrm{a}$ \\
\hline Asturias & & 10 & 0.812 & $\mathrm{a}$ & 10 & 0.142 & $\mathrm{a}$ & 10 & 0.040 & $\mathrm{a}$ & 10 & 0.060 & $\mathrm{a}$ & 6 & 0.005 & $\mathrm{a}$ & 10 & 11.0 & $\mathrm{a}$ & 10 & 7.9 & $\mathrm{a}$ & 10 & 17.6 & $\mathrm{a}$ & 10 & 56.0 & $\mathrm{a}$ \\
\hline Bierzo & & 10 & 0.959 & $\mathrm{~b}$ & 10 & 0.277 & b & 10 & 0.035 & a & 10 & 0.068 & $a b$ & 10 & 0.004 & $\mathrm{a}$ & 10 & 12.5 & a & 10 & 6.8 & $\mathrm{a}$ & 10 & 18.8 & a & 10 & 33.1 & $\mathrm{a}$ \\
\hline Extremadura & & 2 & 0.800 & $\mathrm{a}$ & 2 & 0.110 & $\mathrm{a}$ & 2 & 0.035 & $\mathrm{a}$ & 2 & 0.067 & $a b$ & & - & & 2 & 12.0 & $\mathrm{a}$ & 2 & 8.1 & $\mathrm{a}$ & 2 & 15.5 & $\mathrm{a}$ & 2 & 23.0 & $\mathrm{a}$ \\
\hline Galicia & & 36 & 0.904 & $a b$ & 36 & 0.192 & $\mathrm{a}$ & 36 & 0.044 & $\mathrm{a}$ & 36 & 0.067 & $a b$ & 30 & 0.011 & a & 36 & 12.8 & $\mathrm{a}$ & 36 & 6.9 & $\mathrm{a}$ & 36 & 18.7 & $\mathrm{a}$ & 36 & 34.5 & $\mathrm{a}$ \\
\hline Spain & & 71 & 0.900 & & 71 & 0.188 & & 71 & 0.042 & & 71 & 0.068 & & 57 & 0.008 & & 71 & 12.3 & & 71 & 7.1 & & 71 & 18.1 & & 71 & 38.7 & \\
\hline
\end{tabular}

ns: non significant.

${ }^{\text {a }}$ For each column, means with different letters are significantly different $(P=0.05)$ according to Student-Newman-Keuls multiple range test.

* Significant at $P \leq 0.05$, respectively.

***** Significant at $P \leq 0.01$, respectively.

Significant at $P \leq 0.0001$, respectively. 
pure species $50 \%$ for C. dentata, $49 \%$ for $C$. mollissima and $40 \%$ for $C$. sativa (data refers to dry matter). Significantly lower starch content was found in cultivars from Canary Islands in comparison with the other regions $(46 \%)$ where it was Redonda which showed the lowest starch content (42\%). Highest average starch content per region was found in Galician cultivars $(60 \%)$, where the cultivar Longal showed the highest value (67\%); a higher value than previously reported by Ferreira-Cardoso et al. (1993) for the Portuguese Longal (53-55\%). This higher starch content should be taken into account in selecting cultivars for flour production or for animal feed. Starch is partially hydrolysed into total sugars, which gives sweetness to chestnuts (Table 2). The Pearson correlation between starch and total sugars was very high, $-0.88(P<0.0001)$. Canary Islands showed highest and significant total sugar content of $21 \%$, and Andalucía, Extremadura and Galicia the lowest with 14\%, the average for Spain being $15 \%$. The long held myth by growers that smaller nuts are sweeter has not been observed in the current results with a low correlation between total sugar content and nut weight.

Crude fibre did not show significant differences between regions or cultivars within regions and agreed with the results of acid detergent fibre and neutral detergent fibre showing the highest values for chestnuts from Andalucía, 2.9\%, and the lowest from Extremadura, 2.5\% (Table 3). Our values of crude fibre were higher than those obtained by McCarthy and Meredith (1988) for European chestnut (1.4\%), Chinese (1.6\%) and American (1.9\%). Chestnuts show similar contents of crude fibre to other nuts; walnuts $(2.1 \%)$, pecans $(2.3 \%)$ and pistachios $(1.9 \%)$ but smaller than hazelnuts $(6.1 \%)$ (Ensminger et al., 1995). Nuts are generally recommended, with vegetables, fruits and cereal products, to increase the fibre in the diet (Ensminger et al., 1995), although it can reduce the interest in respect of some cultivars for animal feed.

When the crude fibre system was developed, it was thought to represent most of the cell wall portion of the forage. However, it was later discovered that it had not accounted for some of the hemicellulose and lignin components. The fibre detergent system was used for determining the cell wall matrix and estimating its major subcomponents: hemicellulose, cellulose and lignin. The ADF residue recovers lignin, cellulose and the least digestible noncarbohydrate fractions and is widely used as a quick estimation of fibre in feeds, often substituting for crude fibre as part of a proximate analysis (Van Soest, 1994). ADF was correlated with crude fibre in our work (0.64, $P<0.0001)$. The average value for Spain was $3 \%$ with a significantly low value of 2.7-2.8 for Extremadura and Galicia and a maximum of $3.5 \%$ in Andalucía (Table 3).

Neutral detergent fibre fraction is defined by Van Soest (1994) as the percentage of cell wall material or plant structure in a feed. NDF recovers the major cell wall components lignin, cellulose and hemicellulose, and also contains minor cell wall components, including some protein and bound nitrogen, minerals and cuticle but pectins are removed. Normally, NDF is inversely related to intake, therefore, a low percentage of NDF is desirable to get a good intestine digestion. No significant differences were found between regions. The lowest value corresponded for Extremadura with $16 \%$, the same as in Asturias, being the highest in Andalucía with $18 \%$ (Table 3). Strong differences between cultivars were found, $9.4 \%$ for Capilla and $28.5 \%$ for Pilonga, both from Andalucía.

Fat content in chestnuts is low but of high quality with a value 2-3\% according to Breisch (Breisch, 1995), McCarthy and Meredith (1988) and Ensminger et al. (1995), in comparison with other nuts such as almonds $(53.9 \%)$, hazelnuts $(35.9 \%)$ or walnuts $(63.4 \%)$. For Spanish chestnuts, the average value was $3.0 \%$, with a range from $2.8 \%$ in Extremadura to $3.2 \%$ in Asturias (Table 3). Significant differences were found between cultivars, with the lowest value for Llanisca from Asturias, $1.7 \%$, and the highest for Pilonga and Parede from Andalucía and Galicia, respectively, both with $4.0 \%$.

No significant differences were found in crude protein content between regions or cultivars, the average value of $5.8 \%$ in Spain (Table 3), much higher than the $2.9 \%$ reported by Ensminger et al. (1995) and similar to the values between 3 and $6 \%$ showed by Breisch (1995). This protein content is lower than other nuts such as almonds (19\%) or walnuts $(21 \%)$ and legumes (over $20 \%$ ) (Ensminger et al., 1995). Between cultivars, Chamberga showed the lowest value with $4.5 \%$ and the highest by Dieguina with $9.6 \%$. Our results did not reveal any relationship between protein content of chestnuts and soil type as reported by Gomes et al. (1997) and De La Montaña Míguelez et al. (2004).

Ash content is low in chestnuts, between 1.8 (cv. Injerta, Extremadura) and 3.2\% (cv. Dieguina, Andalucía), with an average value of $2.3 \%$ for Spain (Table 3 ). These values were higher than those reported by McCarthy and Meredith (1988) and Breisch (1995).

\subsection{Mineral content}

Breisch (1995) indicated that the main macrominerals are Potassium, Phosphorus, Calcium and Magnesium and our results also agree with an average for Spain of $0.900 \%$ (from $0.789 \%$ for cv. Parede from Galicia to $1.130 \%$ for cv. Dieguina from Andalucía), $0.188 \%$ (from $0.110 \%$ for cv. Verata from Extremadura to $0.305 \%$ for cv. Inxerta from Galicia), $0.042 \%$ (from $0.026 \%$ for cv. Longal to $0.072 \%$ for cv. Verde, both in Galicia) and $0.068 \%$ (from $0.049 \%$ for cv. Longal from Galicia to $0.100 \%$ for cv. Dieguina from Andalucía), respectively (Table 4). Average level for Sodium in Spain has been $0.008 \%$ with a minimum of $0.003 \%$ for cv. Negral, from Galicia and El Bierzo, cv. Helechal from Andalucía and cv. Doriga from Asturias, and a maximum of $0.024 \%$ for cv. Famosa from Galicia.

For the microelements, Manganese was the most important mineral with $38.7 \mathrm{ppm}$ on average for Spanish chestnuts, followed by Iron, $18.1 \mathrm{ppm}$; Zinc, $12.3 \mathrm{ppm}$; and Copper, $7.1 \mathrm{ppm}$ (Table 4). Significant differences were found between cultivars in Manganese content with a range from $17.0 \mathrm{ppm}$ (cv. Rapada, Castilla-León) to 124 ppm (cv. Llanisca, Asturias). All values were higher than those reported by Breisch (1995) and it is as good a source of Manganese as wheat or peanut (Ensminger et al., 1995).

No significant differences were found between regions and cultivars for Iron with $18.1 \mathrm{ppm}$ (from $14.0 \mathrm{ppm}$ for cv. Inxerta 


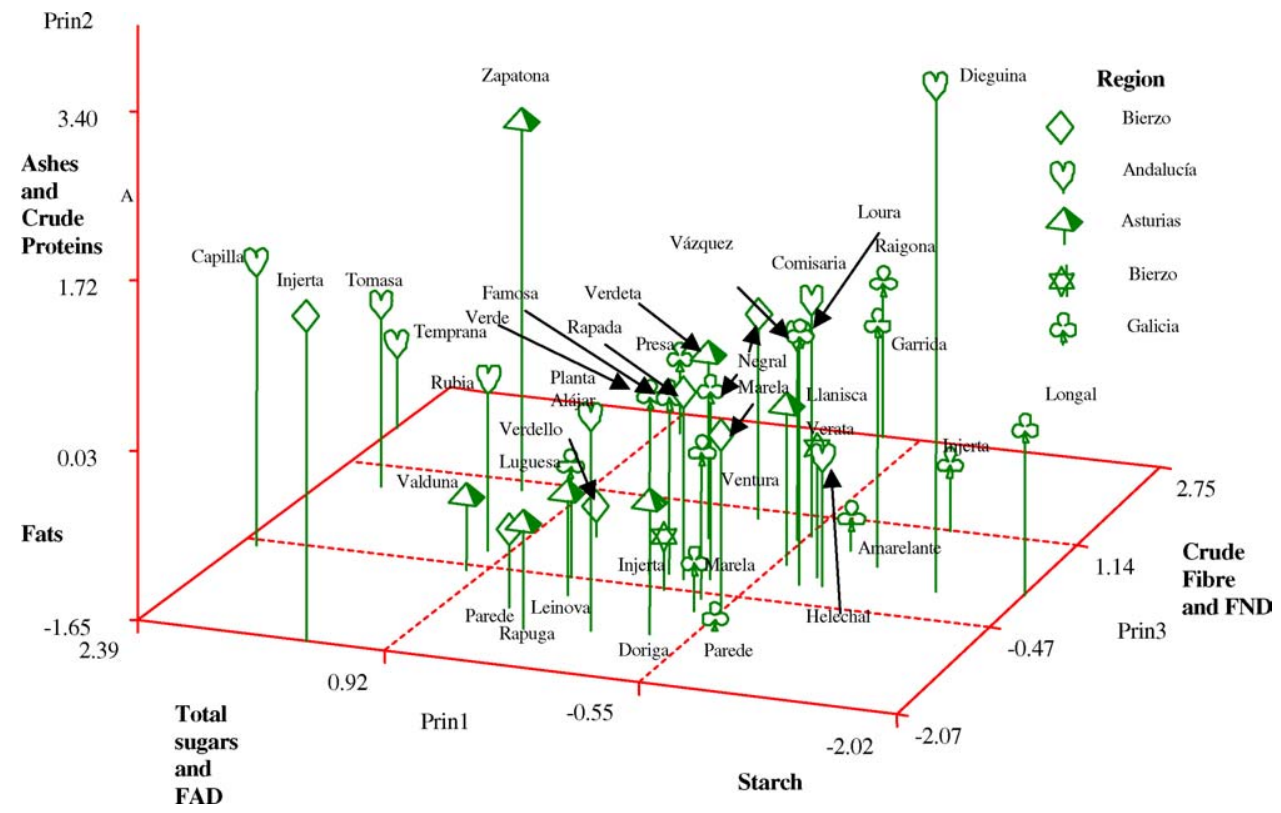

Fig. 2. Variability of the most important chestnut cultivars in Spain using the first principal components.

from Galicia to $23.8 \mathrm{ppm}$ for cv. Doriga from Asturias), Zinc with $12.3 \mathrm{ppm}$ (from $10 \mathrm{ppm}$ for cv. Verdello from CastillaLeón to $19 \mathrm{ppm}$ for cv. Verde from Galicia), and Copper with $7.1 \mathrm{ppm}$ (from $5.5 \mathrm{ppm}$ for cv. Garrida from Galicia to $10.3 \mathrm{ppm}$ for cv. Doriga from Asturias). Iron values were lower that those showed by Breisch (1995) and closer to Ensminger et al. (1995) and those values reported by McCarthy and Meredith (1988) for American and Chinese chestnuts. Zinc values were double to those reported by Breisch (1995) and Ensminger et al. (1995) and similar to American and Chinese nuts (McCarthy and Meredith, 1995). Copper contents were lower than those found by Breisch (1995) and Ensminger et al. (1995) and higher than those reported by McCarthy and Meredith (1988) for American and Chinese chestnuts. Correlations between minerals and environment were low and not significant, indicating that differences founds between cultivars were due to genetic differences.

\subsection{Classification by multivariate analysis}

Using principal component analysis to classify cultivars and regions according to the main nutrient components, we have found that the three main principal components with 18 values over 1 , accumulated more than $70 \%$ of the variance. First principal component (PC) discriminated positively to cultivars with high contents of soluble sugars and ADF and negatively the cultivars with lower starch content. Second PC scores cultivars with high ash and protein contents positively and high fat content negatively. Third PC scatters positively cultivars with high crude fibre contents and negatively those with high sugar content. Cultivars such as Capilla, Temprana and Tomasa from Andalucía showed the highest total sugar contents (positive PC1), and Dieguina a lower value (negative PC1) combined with a high content of ashes and proteins (PC2 positively, Fig. 2). In Asturias, Zapatona was the most unusual cultivar with very high content of ashes and protein (positive PC2). Cultivars from Galicia showed the highest starch content (negative PC1) in Longal, Injerta and Garrida. Cultivar Injerta from Castilla-León (El Bierzo) had the highest content of soluble sugars (positive PC1) and as well as for ashes and proteins (positive PC2).

\section{Conclusions}

The variabilities found in nutrient composition between cultivars and between regions correspond to the high genetic diversity found between cultivars, previously verified by morphology and molecular markers (Ramos-Cabrer and Pereira-Lorenzo, in press). This variability in nutrients can be useful for selecting specific cultivars for the market, such as flour production. For example, breeding programs with cultivars Longal, Inxerta and Garrida are suited to industry purposes due to their high starch contents. Cultivars as Pilonga, Temprana and Capilla presented low moisture contents that reduce their suitability for fresh market conservation. In addition, cv. Pilonga showed high fibre content that reduces its digestibility and makes it unfavorable to the consumer.

\section{Acknowledgments}

This work is funded by INIA and MCYT (projects FOA97165, SC99-036-C2-23 and AGL2003-09874-C02-01).

We thank to Dr. Yoong Lim (Queen Mary College, University of London, UK), Dr. Begoña de la Roza-Delgado (Área de Nutrición, Pastos y Forrajes, SERIDA, Spain) and Dr. Antonio Bentabol-Manzanares (Jefe de la Unidad de Valorización Agroalimentaria del Cabildo de Tenerife, Tenerife, Spain) for reviewing this paper. 


\section{References}

AOAC, 1984. In: Horwitz, W. (Ed.), Official Methods of Analysis. 14th ed. AOAC, Washington, DC.

AOAC, 1990. In: Horwitz, W. (Ed.), Official Methods of Analysis. 15th ed. AOAC, Washington, DC.

B.O.E., 1995. R.D. 2257/1994 de 25-II-94. B.O.E. no. 52 de 02/03/1995, 52, 7161-7237.

B.O.E., 2000. OM 3195 de 16-02-2000. B.O.E. no. 41 de 17/02/2000, Anexo 34 (a).

Breisch, H., 1995. Châtaignes et marrons. CTIFL, Paris.

De La Montaña Míguelez, J., Míguez Bernárdez, M., García Queijeiro, J.M., 2004. Composition of varieties of chestnuts from Galicia (Spain). Food Chem. 84, 401-404.

Ensminger, A.H., Ensminger, M.E., Konlande, J.E., Robson, J.R.K., 1995. The Concise Encyclopedia of Foods and Nutrition, second ed. CRC Press, BocaRaton.

Ferreira-Cardoso, J.V., Fontainhas-Fernandes, A.A., Torres-Pereira, J.M.G., 1993. Nutritive value and technological characteristics of Castanea sativa Mill. Fruits-Comparative study of some Northeastern Portugal cultivars. In: Istituto di Coltivazioni Arboree University of Perugia, Perugia (Ed.), Proceedings of the International Congress on Chestnut Spoleto, Comunità Montana Monti Martani e Serano of Spoleto, Istituto di Coltivazioni Arboree University of Perugia, Italy, pp. 445-449.

Gomes, A.L., Abreu, C.G., Castro, L.T., 1997. COLUTAD Um clone de castanheiro com resistência à donça da tinta. NATO/SFS Programme III PO-Chestnut. Vila Real, Universidade de Tras os MOntes e Alto Douro.

McCarthy, M.A., Meredith, F.I., 1988. Nutrient data on chestnuts consumed in the United States. Econ. Bot. 42 (1), 29-36.

Pereira-Lorenzo, S., Díaz-Hernandez, B., Ciordia-Ara, M., Ascasibar-Errasti, J., Ramos-Cabrer, A.M., Sau, F., 2001a. Spanish chestnut cultivars. HortScience 36 (2), 344-347.

Pereira-Lorenzo, S., Ramos-Cabrer, A.M., Rios, D., Perdomo, A., GonzálezPérez, J., 2001b. Update of the Spanish chestnut inventory of cultivars. FAOCIHEAM-Nucis-Newslett. 10, 34-37.
Pereira-Lorenzo, S., Ríos, D., González-Pérez, J., Cubas, F., Perdomo, A., Calzadilla, C., Ramos-Cabrer, A.M., 2001c. Chestnut cultivars on the Canary Islands. For. Snow Landsc. Res. 76 (3), 445-450.

Pereira-Lorenzo, S., Fernández-López, J., Moreno-González, J., 1996a. Variability and grouping of Northwestern Spanish Chestnut Cultivars (Castanea sativa). I. Morphological traits. J. Am. Soc. Hort. Sci. 121 (2), 183-189.

Pereira-Lorenzo, S., Fernández-López, J., Moreno-González, J., 1996b. Variability and grouping of Northwestern Spanish Chestnut Cultivars. II. Isoenzyme traits. J. Am. Soc. Hort. Sci. 121 (2), 190-197.

Pereira-Lorenzo, S., Ramos-Cabrer, A.M., 2003. Características morfológicas e isoenzimáticas de los cultivares de castaño (Castanea sativa Mill.) de Andalucía, vol. 13. Monografías INIA, Agrícola, 160 pp.

Pereira-Lorenzo, S., Ramos-Cabrer, A.M., Díaz-Hernández, M.B., Ciordia-Ara, M., 2005. Características morfológicas e isoenzimáticas de los cultivares de castaño (Castanea sativa Mill.) de Asturias, vol. 16. Monografías INIA, Serie Agrícola, 541 pp.

Pereira-Lorenzo, S., Ramos-Cabrer, A.M., 2004. Chestnut, an ancient crop with future. In: Ramdane, D., Mohan-Jain, S. (Eds.), Production Practices and Quality Assessment of Food Crops, vol. 1 "Preharvest Practice" Kluwer Academic Publishers, Dordrecht, The Netherlands, pp. 105-161.

Ramos-Cabrer, A.M., Pereira-Taboada, A., Pereira-Lorenzo, S., 2003. Características morfológicas e isoenzimáticas de los principales cultivares de castaño Castanea sativa Mill. de El Bierzo (Castilla y León) y Guadalupe (Extremadura), vol. 14. Monografías INIA, Agrícola, 103 pp.

Ramos-Cabrer, A.M., Pereira-Lorenzo, S., in press. Genetic relationship between Castanea sativa Mill. trees from North-western to South Spain based on morphological traits and isoenzymes. Genetic Resources and Crop Evolution, in press.

SAS/STAT, 1988. User's Guide, Release 6.03 Edition. SAS Institute Inc., Cary, p. 13.

Van Soest, P.J., Robertson, J.B., Lewis, B.A., 1991. Methods for dietary fibre, neutral detergent fibre and nonstarch polysacharides in relation to Animal Nutrition. J. Dairy Sci. 74, 3583-3597.

Van Soest, P.J., 1994. Nutritional Ecology of the Ruminant. Cornell University Press, New York. 